Service social

\title{
Les demandeurs d'asile au Canada : quelques enjeux pour la pratique du travail social
}

\section{Marie Lacroix}

Volume 51, numéro 1, 2004

URI : https://id.erudit.org/iderudit/012711ar

DOI : https://doi.org/10.7202/012711ar

Aller au sommaire du numéro

Éditeur(s)

École de service social de l'Université Laval

ISSN

1708-1734 (numérique)

Découvrir la revue

Citer cet article

Lacroix, M. (2004). Les demandeurs d'asile au Canada : quelques enjeux pour la pratique du travail social. Service social, 51(1), 45-59.

https://doi.org/10.7202/012711ar
Résumé de l'article

Cet article s'intéresse au sort des demandeurs d'asile au moment où ils se réinstallent en cultivant l'espoir de s'intégrer un jour à la société canadienne. Il porte aussi sur certains des principaux éléments du processus de détermination du statut de réfugié qui se sont avérés des obstacles à l'intégration à long terme à la société d'accueil. Dans ce contexte, les habiletés et les connaissances particulières à chacune des cultures constituent un niveau d'intervention auprès des demandeurs d'asile. Cependant, en plus d'être conscients des enjeux propres à la culture et au pays d'origine, les intervenants doivent saisir le sens de la migration. La migration, qu'elle soit volontaire (immigrants) ou forcée (réfugiés et demandeurs d'asile), constitue une expérience douloureuse qui comporte toujours une rupture avec le passé. Le travail social peut jouer un rôle fondamental dans un processus qui sert à retisser les liens sociaux. 


\title{
Les demandeurs d'asile au Canada : quelques enjeux pour la pratique du travail social
}

\author{
Marie LACROIX \\ Professeure adjointe \\ École de service social \\ Université de Montréal
}

Cet article s'intéresse au sort des demandeurs d'asile au moment où ils se réinstallent en cultivant l'espoir de s'intégrer un jour à la société canadienne. II porte aussi sur certains des principaux éléments du processus de détermination du statut de réfugié qui se sont avérés des obstacles à l'intégration à long terme à la société d'accueil. Dans ce contexte, les habiletés et les connaissances particulières à chacune des cultures constituent un niveau d'intervention auprès des demandeurs d'asile. Cependant, en plus d'être conscients des enjeux propres à la culture et au pays d'origine, les intervenants doivent saisir le sens de la migration. La migration, qu'elle soit volontaire (immigrants) ou forcée (réfugiés et demandeurs d'asile), constitue une expérience douloureuse qui comporte toujours une rupture avec le passé. Le travail social peut jouer un rôle fondamental dans un processus qui sert à retisser les liens sociaux.

Mots clés : demandeurs d'asile, intégration, processus décisionnel du statut de réfugié, réfugiés.

This article addresses the plight of asylum seekers as they resettle and entertain the hope of eventually integrating into Canadian society. Are addressed some of the major elements of the refugee determination process that have been shown to present obstacles to long term integration into the host society. In this context, culturally specific knowledge and skills constitute one level of intervention with asylum seekers. However, in addition to being aware of issues related to culture and country of origin, practitioners must understand the meaning of migration. Whether voluntary (immigrants) or involuntary (asylum seekers and refugees), migration is a painful experience and always involves a rupture with the past. Social work can be instrumental in a process that serves to rebuild social bonds.

Keywords: asylum seekers, integration, refugee determination process, refugees. 


\section{INTRODUCTION}

Au Québec, l'intervention interculturelle a été l'approche dominante dans le travail auprès des immigrants et des réfugiés. Bien que l'on ait tenu compte dans cette approche de certains enjeux propres à la migration, la compréhension de l'incidence de la migration est demeurée, somme toute, marginale. Maintenant que le domaine de la migration est abordé en soi (Soydan, 1998), de nouveaux champs de pratique et de recherche s'ouvrent au travail social. Ainsi, les praticiens fonctionnent dorénavant selon différents scénarios de réinstallation et d'intégration des immigrants et des réfugiés (Valtonen, 2004). L'un de ces scénarios inclut les demandeurs d'asile qui, à cause du caractère précaire de leur statut, se retrouvent pris dans un système complexe qui aura des conséquences tragiques sur leurs vies ainsi que sur leur possibilité de demeurer au Canada et de s'y installer de façon permanente.

Les demandeurs d'asile entrent au pays avec un statut précaire qui en fait un groupe d'individus particulièrement vulnérables. On a accordé peu d'attention à l'incidence d'un vécu à statut précaire et des études récentes indiquent que le statut au regard de l'immigration a une incidence importante sur la vulnérabilité des réfugiés (Cambridge et Williams, 2004) et qu'il est important d'intégrer au processus d'intégration à long terme des demandeurs d'asile l'expérience qu'ils ont vécue au moment du processus de revendication du statut de réfugié (Mestheneos, Ioannidi-Kapolou et Gaunt, 1999; Mestheneos et Ioannidi, 2002; CERE, 1999). De plus, la recherche actuelle indique que l'« intégration » à long terme à la société d'accueil s'amorce dès l'arrivée. Pour cette raison, on devrait se préoccuper lors de toute intervention d'établir des conditions qui faciliteront l'installation à long terme.

La manière dont la société d'accueil reçoit les demandeurs d'asile à l'aide de politiques et de pratiques et la façon dont les praticiens de première ligne répondent à leurs besoins au moment du premier contact avec la société d'accueil auront une incidence sur leur capacité de s'installer à long terme. Les travailleurs sociaux sont souvent les premiers à rencontrer les demandeurs d'asile une fois qu'ils sont entrés au pays. Leur capacité d'accueillir les demandeurs d'asile et de répondre aux besoins inhérents à leur situation constituent donc des éléments cruciaux de la phase d'accueil.

Cet article examine certains des principaux éléments du processus décisionnel du statut de réfugié qui se sont avérés des obstacles à l'intégration à long terme à la société d'accueil. La première partie de cet article définit quelques-uns des concepts importants, puis on y aborde certains des principaux enjeux propres à l'intervention auprès d'une population qui vit avec un statut précaire.

\section{RÉFUGIÉS AU SENS DE LA CONVENTION}

Un demandeur d'asile est une personne qui revendique le statut de réfugié une fois dans le pays, soit à la frontière, soit à un bureau d'immigration à l'intérieur du pays. Le demandeur devra démontrer qu'il est un réfugié tel que le définit la Convention de 
Genève. La Convention de Genève (ONU) relative au statut des réfugiés (1951) et le Protocole de 1967 définissent le réfugié comme étant un individu qui :

en raison d'une crainte justifiée de persécution du fait de sa race, de sa religion, de sa nationalité, de son appartenance à un certain groupe social ou de ses opinions politiques

i) se trouve hors du pays dont il a la nationalité et ne peut ou, du fait de cette crainte, ne veut se réclamer de la protection de ce pays ou,

ii) n'ayant ni pays ni nationalité et se trouvant hors du pays dans lequel il avait sa résidence habituelle ne peut ou, en raison de ladite crainte, ne veut y retourner.

L'intervention auprès des demandeurs d'asile doit tenir compte de tout un éventail d'enjeux, dont la précarité du statut au regard de l'immigration. Étant donné le caractère de ce statut, il est impossible de planifier à long terme comme peuvent le faire les réfugiés sélectionnés à l'étranger ou les immigrants qui arrivent munis du statut de résident permanent. Bien que le statut de demandeur d'asile soit temporaire et que la demande du requérant puisse être rejetée, environ 40 à $45 \%$ des demandes seront acceptées. II importe de signaler que les demandeurs d'asile arrivent en sollicitant la protection du Canada et que leur but à long terme est de s'installer dans ce pays et de s'intégrer un jour à la société d'accueil. Cependant, l'intégration est un projet à long terme et, pour ce qui est des demandeurs d'asile, elle doit être perçue comme un processus à plusieurs étapes qui s'amorce le jour où la demande d'asile est déposée. Pour une intervention bien planifiée, il faut donc au préalable bien saisir les principaux concepts propres à ce domaine.

\section{INTÉGRATION}

Bien que le concept de l'intégration fasse toujours l'objet d'un débat (pour un excellent aperçu, se reporter à Castles et al., 2003) - ce qu'il signifie, qui le définit et à quelles fins -, nous ferons appel pour cet article à une définition très générale qui met en évidence certains des principaux éléments à prendre en considération lors du processus d'intervention :

L'intégration concerne les immigrés qui se stabilisent sur le territoire et qui, avec le temps et par leurs enfants, finissent par devenir partie intégrante de la société d'accueil (Blanc-Chaléard, 2004).

« Stabiliser » constitue ici le concept important. Dès que la demande d'asile est déposée, il faudra aux demandeurs de l'assistance pour stabiliser leur situation matérielle, sociale et émotionnelle. 


\section{RÉINSTALLATION}

Dès que les demandeurs d'asile arrivent au pays, ils doivent s'installer pendant qu'ils vont de l'avant avec leur demande d'asile. II est important d'être conscient de cette première phase de l'installation qui, selon Valtonen (2004, p. 70), « se rapporte aux activités et processus de se fixer après l'arrivée dans le pays d'installation ». Le contexte de la recherche d'asile suppose que les personnes ont été forcées de quitter leur pays pour des raisons politiques, sociales ou autres qui échappent à leur contrôle. Leur situation est donc différente de celle d'un immigrant qui a planifié librement et de son propre gré de migrer vers un autre pays. Les demandeurs d'asile se retrouvent donc dans une situation de vulnérabilité qu'ils ne contrôlent pas et sont obligés de se débrouiller dans une situation de précarité. Lorsqu'on parle de réfugiés ou de demandeurs d'asile il est pertinent d'utiliser le concept de réinstallation qui « admet le fait que les réfugiés étaient déjà "installés" dans leur pays d'origine en matière de qualifications, d'emploi, de statut social, ainsi que de réseaux sociaux et familiaux, etc. » (Castles et al., 2003). Bien comprendre la rupture avec leur vie passée est un important élément structurel à prendre en considération lors de l'intervention; cela permet d'entrer en relation d'aide avec des gens que l'on peut percevoir comme temporairement dépourvus des moyens de gérer une situation complexe. En effet, comme nous l'avons déjà démontré (Lacroix, 2000), les réfugiés ne sont pas simplement l'étiquette que leur attribue la loi de l'immigration. II s'agit de personnes qui ont un passé, une histoire et un patrimoine culturel.

\section{LA PRÉCARITÉ DE STATUT}

La Loi sur l'immigration et la protection des réfugiés canadienne de 2002 prévoit un certain nombre de catégories d'immigrants qui sont de nature précaire (c'est-à-dire que l'on pourrait leur refuser le droit de demeurer au Canada) et qui dépendent de tierces parties: les demandeurs d'asile, les membres parrainés de la catégorie du regroupement familial, les détenteurs de permis de travail temporaire et les aides familiales résidantes (Oxman-Martinez et Lapierre-Vincent, 2002). Pendant qu'ils sont en attente d'un statut, les demandeurs d'asile sont très conscients de la possibilité que leur demande d'asile leur soit refusée et qu'ils peuvent par conséquent perdre le droit de demeurer au Canada. Cela supposerait la déportation vers le pays des persécutions dénoncées ou vers un pays tiers par lequel ils ont transité pour se rendre au Canada. Quoi qu'il en soit, ces possibilités suscitent un stress, des craintes et des inquiétudes parfois exaspérantes chez les requérants (Lacroix, 2004; Sourander, 2003).

\section{LE PROCESSUS DE REVENDICATION DU STATUT DE RÉFUGIÉ}

Tous les demandeurs d'asile qui déposent une demande de statut de réfugié n'obtiendront pas la permission de demeurer au Canada. Toutefois, environ la moitié d'entre eux seront reconnus comme réfugiés au sens de la Convention et deviendront résidents permanents. De janvier à juin 2004, 12207 demandes ont été déposées et 
30468 personnes étaient en attente d'une décision. Selon les statistiques de la Commission de l'immigration et du statut de réfugié, le taux d'acceptation global a alors été de $40 \%$ (Conseil canadien pour les réfugiés, 11 août 2004). Ce chiffre peut varier selon les situations politiques qui ont cours dans les divers pays d'où viennent les réfugiés; par exemple, $79 \%$ des demandes d'asile présentées par des ressortissants du Zimbabwe au cours de l'année 2002 ont été acceptées. Au Canada, le processus décisionnel du statut de réfugié est long et complexe. Lorsqu'ils déposent leur demande, les demandeurs reçoivent des formulaires qu'ils doivent remplir et renvoyer à la Commission de l'immigration et du statut de réfugié (CISR) dans les 28 jours. Le demandeur attend alors qu'on fixe une date d'audience à la CISR. Après l'audience, il doit attendre la réponse. Si la décision est positive, le demandeur se voit octroyer le droit de déposer une demande de résidence permanente, ce qui amorce immédiatement le processus de la réunification des familles. Si la décision est négative, le demandeur peut demander l'autorisation d'en appeler auprès de la Cour fédérale ou demander un réexamen de son cas pour considérations humanitaires. Si ces recours ne réussissent pas, une mesure d'exclusion sera décrétée, ce qui empêche toute possibilité de résidence permanente, et l'on se préparera à la déportation du demandeur d'asile.

Il a été démontré que, dans la plupart des pays occidentaux, le processus décisionnel du statut de réfugié est ardu et l'on a établi que la longueur de la période que dure ce processus constitue un obstacle important au processus d'intégration (Drozdek et al., 2003; Mestheneos et Ioannidi, 1999; CERE, 1999; Lacroix, 2000; Rousseau et al., 2002). Bien que certains des plus importants facteurs relevés par des chercheurs par le passé soient analysés en tant qu'obstacles à l'intégration, ils peuvent aussi servir à l'évaluation du parcours du demandeur d'asile et à structurer un plan d'intervention. II s'agit de :

\section{LA QUALITÉ DE L'ÉTAPE DE LA RÉCEPTION}

Le premier contact avec la société d'accueil se produit par le biais du processus décisionnel du statut de réfugié. Cette étape s'amorce dès l'accueil à la frontière ou à l'endroit où le migrant dépose sa demande d'asile. Une bonne part de cette première partie du processus entraîne des rencontres avec les agents de l'immigration et est vécue avec angoisse et appréhension. À cette étape, les demandeurs d'asile doivent se conformer à un nouvel ensemble de règles, ce qui suscite de l'anxiété chez eux. Ils ne savent ni quoi attendre du processus, ni ce que l'on attend d'eux. Chez ceux qui ont voyagé munis de faux passeports, l'angoisse est d'autant plus grande qu'ils savent qu'ils ont menti et ils redoutent la façon dont cela sera perçu par les agents de l'immigration.

La première rencontre avec un agent de l'immigration suscitera de nombreuses questions qui restent souvent sans réponse, tant que le demandeur n'a pas trouvé les ressources adéquates pour y répondre. La manière dont le demandeur est accueilli, la façon dont on répond aux questions, la qualité de l'information reçue, l'accès à une 
première orientation et aux premiers services pour le guider dans la société d'accueil font partie des principales questions qu'il faut aborder en intervention.

\section{LA LONGUEUR DE LA PÉRIODE D'ATTENTE}

Des recherches universitaires récentes confirment les connaissances pragmatiques acquises par ceux qui œuvrent auprès des réfugiés quant aux carences du système (Rousseau et al., 2002; Barsky, 2000). Quoique certaines demandes d'asile puissent être dirigées vers un système accéléré qui comporte un délai de décision de quelques mois, les recherches indiquent que, pour plusieurs demandeurs d'asile au Québec, la période d'attente moyenne d'une audience est de 7 mois et qu'elle est de 22 mois pour le traitement de leur demande de résidence permanente (Renaud et Gingras, 1998). Au cours de cette période, les demandeurs d'asile ont un accès restreint au travail, à l'aide sociale ainsi qu'aux services sociaux et aux soins de santé. L'attente constitue peut-être l'aspect le plus pénible du processus, puisque leurs vies sont mises en suspens et qu'on les pousse dans une situation dont l'issue est incertaine (Lacroix, 2000).

\section{ACCÈS À DES SERVICES POUR L'INSTALLATION}

Pour faciliter leur installation, les demandeurs d'asile ont besoin d'orientation et de services. Au Québec, les services accessibles aux demandeurs d'asile se limitent à la recherche de logement. Bien que certains organismes communautaires procurent information et orientation vers les services pour lesquels ils ne sont pas subventionnés par la province (Bertot et Mekki-Berrada, sept. 1999), les demandeurs d'asile sont pour une large part laissés à eux-mêmes durant le processus décisionnel du statut de réfugié. La recherche universitaire constate « le besoin d'un guichet unique où les demandeurs d'asile pourraient accéder à de l'information, à des conseils et à de l'orientation pour un éventail de questions » (Aldridge et Waddington, 1999, p. 19).

Une équipe de recherche de Montréal constate que :

en n'offrant pas davantage de services à ces milliers de personnes vulnérables et en voie de devenir des citoyens à part entière, celles-ci vivent des difficultés postmigratoires qui viennent potentialiser les effets négatifs des traumatismes prémigratoires (violence organisée, torture, viol, emprisonnement, menaces, etc.) (Bertot et Mekki-Berrada, sept. 1999).

Ainsi, les décideurs politiques, en négligeant l'importance sociale de services pertinents pour les demandeurs d'asile, sapent eux-mêmes leur propre projet de société qui consiste à favoriser l'intégration (ibid.).

\section{LA SÉPARATION DE FAMILLES}

Certains effets secondaires de la politique relative à l'immigration, notamment la séparation des familles, alimentent un processus de marginalisation persistante et peuvent engendrer des traumatismes psychologiques graves, la dépression, l'éclatement des familles, des difficultés à l'intégration et des difficultés financières (Rousseau et al., 1997). Bertot et Mekki-Berrada (1999) indiquent que $80 \%$ des demandeurs d'asile 
arrivent sans leur famille immédiate, que $62 \%$ sont loin de l'ensemble de leur famille et que $18 \%$ d'entre eux sont séparés de certains membres de leur famille (époux ou épouse et enfants de moins de 19 ans).

Alors que les hommes se déplacent seuls, les femmes et les enfants sont souvent laissés derrière, parfois cachés dans leur pays d'origine, parfois dans un pays tiers où ils ne disposent plus de la protection de leur mari ou de leur père et n'ont plus les moyens financiers d'assurer leur survie. Cette situation est à la source d'une inquiétude accablante pour le demandeur d'asile qui se trouve au Canada et qui tente d'assumer son rôle de pourvoyeur pour sa famille, tout en se débrouillant tant bien que mal dans un contexte où il est dépossédé de son pouvoir d'action sur son propre sort.

\section{QUALIFICATION PROFESSIONNELLE ET EMPLOI}

Les difficultés liées à l'accès au travail, le sous-emploi (Beiser et Feng-Hou, 2001) et l'absence de reconnaissance des titres de compétence ont été considérés comme des éléments importants qui nuisent au processus d'intégration des réfugiés (Austin et Este, 2001; Krahn et al., 2000; Li, 2001; Aldridge et Waddington, 2001). L'impossibilité de travailler ou l'accès qui se limite à certains domaines de travail poussent les demandeurs d'asile à demander de l'aide sociale (Bloch et Schuster, 2002; Lacroix, 2000; Marr et Siklos, 2001). À long terme, cette situation peut mener à leur marginalisation économique et à une absence d'occasion de participer au marché du travail (Kazemipur et Halli, 2001) d'une façon qui leur soit significative et qui permette une réelle participation citoyenne.

Renaud et Gingras (1998) ont constaté que près de la moitié des réfugiés au sens de la Convention de leur étude n'avaient pas encore obtenu un premier emploi après trois ans. À peine trois demandeurs d'asile sur dix auraient travaillé chaque mois. Le revenu de travail moyen était de 290 \$ par semaine dans quatre principaux secteurs industriels - la fabrication autre que le textile, l'industrie du vêtement, la restauration et d'autres secteurs de service. Sans travail, $90 \%$ d'entre eux recourent à l'aide sociale au troisième mois suivant le dépôt de leur demande. Ce chiffre descend à $44 \%$ au bout de trente-six mois (Renaud et Gingras, 1998, p. 92).

\section{LE LOGEMENT}

Les dimensions relatives au logement du vécu de l'immigrant, notamment l'accès à prix abordable à un logement adéquat et sûr, ont aussi été relevées comme éléments ayant une incidence sur l'intégration (Rose et Ray, 2002; Danso et Grant, 2000). II convient de signaler que les demandeurs d'asile ne sont pas admissibles à des logements subventionnés par les gouvernements ni à des allocations de logement, malgré le fait que leur situation économique les place souvent dans des situations où ils auraient besoin d'une telle assistance. Comme on sait que le logement est lié à la santé, à l'emploi, à la réussite en éducation, à la sécurité, aux réseaux sociaux et à d'autres facteurs sociaux, le manque d'accès à des logements adéquats peut constituer un important obstacle à l'intégration à long terme. 


\section{MÉDIAS, OPINION PUBLIQUE ET RACISME ASSOCIÉ}

La façon dont on présente les demandeurs d'asile dans les médias et les retombées du discours constituent un autre facteur qui influence le rapport que les travailleurs sociaux établissent avec les demandeurs d'asile. Les médias ont engendré des attitudes et des perceptions négatives (Pickering, 2001), surtout après les événements du 11 septembre 2001, dans le contexte des préoccupations relatives à la sécurité nationale (van Selm, 2003), au terrorisme (Hugo, 2002; Simmons, 2002; Aiken, 2000) et à la sécurité des frontières (Adelman, 2002; Oxman-Martinez et al., 2002). Non seulement ces images et ces discours sont-ils de plus en plus liés à une croissance du racisme (Cheran, 2001; Kundnani, 2001; Richmond, 2001; Beiser et al., 2001), ils peuvent aussi renforcer le manque de confiance de la part des intervenants (Cambridge et Williams, 2004) face à une clientèle qu'ils connaissent mal.

Les demandeurs d'asile sont particulièrement sensibles à l'image dont ils sont l'objet dans les médias, lesquels les présentent comme un groupe de personnes qui abusent du système. Le cas récent de Mohammed Cherfi, un Algérien dont la demande d'asile a été rejetée et qui a cherché refuge dans une église de Québec, illustre bien cette situation. Les policiers de la municipalité ont assailli l'église et ont arrêté Cherfi sous prétexte qu'il avait enfreint ses conditions de libération; ils l'ont ensuite remis entre les mains des agents de l'immigration qui l'ont déporté vers les États-Unis, d'où il était arrivé. Les obstacles au travail se dressent devant les demandeurs d'asile lorsque survient la possibilité de revoir leur cas pour motifs humanitaires. Un journaliste a présenté Mohammed Cherfi comme un individu qui avait reçu de l'aide sociale pendant des années, ce qu'il percevait comme une indication de sa réticence à s'intégrer à la société d'accueil. Or, il n'est pas possible pour une personne à statut si vulnérable de se trouver un emploi signifiant et de penser à s'intégrer sans savoir si on lui accordera la résidence permanente pour des motifs humanitaires.

Étroitement associée au racisme des médias, se trouve la mentalité « sécuritariste » qui prédomine depuis les événements du 11 septembre 2001. Les politiques et pratiques mises en place au nom de la sécurité ont d'importantes conséquences sur la sécurité des demandeurs d'asile. La manière dont chacun des travailleurs sociaux comprend tout cela peut aussi avoir une incidence sur la capacité d'intervenir auprès des demandeurs d'asile en provenance de pays ou de groupes ciblés, comme les Arabes ou les Musulmans. C'est dans ce contexte que les demandeurs d'asile arrivent au Canada pour y demander l'asile. C'est à ce moment que plusieurs des demandeurs d'asile entrent en contact avec des intervenants sociaux. 


\section{INTERVENTION}

\section{AU-DELÀ DE LA « CULTURE »}

Il est important de rappeler que ceux qui sollicitent de l'aide le font à un moment de grands besoins et, parfois, au détriment de leur estime de soi et de leur dignité. Le premier pas du processus d'intervention est de reconnaître le fait que les demandeurs d'asile se trouvent dans une situation d'extrême vulnérabilité. Ils viennent d'arriver dans un nouveau pays que, souvent, ils n'ont pas choisi; ils ont perdu leurs repères sociaux et culturels et ils sont pris dans un processus sur lequel ils n'exercent que peu de contrôle. À cela s'ajoute le fait que l'intervention de travailleurs sociaux peut leur être tout à fait étrangère. Les intervenants doivent reconnaître que le travail social a émergé dans un contexte eurocentrique, au sein d'un paradigme de valeurs, de croyances et d'approches propres aux cultures occidentales où l'intervention menée « porte une référence constante à l'individu qui s'oppose à la base communautariste ou collectiviste de l'autre partie du monde » (Rachédi et Vatz-Laaroussi, 2004, p. 7). Par exemple, la classification des problèmes, l'évaluation et l'intervention individuelle sont des concepts qui se basent sur une perspective occidentale du caractère des problèmes sociaux. Bien qu'il s'agisse de la manière dont les sociétés occidentales abordent les problèmes sociaux, de telles perspectives peuvent être étrangères à des sociétés où «c'est le collectif familial qui représente la base communautaire la plus forte pour les sujets » (ibid.) et où les familles et les communautés participent aux processus de résolution de problèmes.

Comme l'a signalé Legault (2000), les demandeurs d'asile ont très souvent des besoins qui se rapportent à leur « installation ». Les demandes d'aide sont alors très souvent ponctuelles et portent sur des préoccupations pragmatiques : trouver un logement, des vêtements pour l'hiver; où inscrire son enfant à l'école; comment traiter avec son propriétaire, etc. Au début du processus d'intervention, on doit clarifier les attentes mutuelles, à la fois celles du travailleur social et celles du demandeur. Les intervenants doivent clarifier leur rôle et les limites de ce qui est possible dans le contexte de leur pratique, qu'elle soit institutionnelle ou communautaire.

Au sein du processus d'intervention, les travailleurs sociaux doivent aussi porter attention aux zones régies par les traditions culturelles du client. La culture n'est pas statique; elle évolue et se transforme au fil du temps. La culture définit qui nous sommes, nos croyances et nos valeurs, d'où nous venons et comment nous percevons le monde. Il devrait être manifeste que les travailleurs sociaux ne rencontrent jamais des « cultures » ou des « groupes ethniques », mais qu'ils rencontrent des personnes qui peuvent se définir ou non selon la manière dont nous les percevons dans un contexte donné. D'autres questions, par exemple le sexe, la classe ou le statut d'immigrant, peuvent prédominer sur des questions de culture ou d'ethnicité dans une situation particulière. Comme nous l'avons démontré dans des recherches antérieures (Lacroix, $2000 ; 2004)$, le vécu des réfugiés se caractérise par le déracinement et le franchissement de frontières. Peu importe leur pays d'origine, on retrouve une expérience commune puisque tous les demandeurs d'asile subissent le processus décisionnel du 
statut de réfugié. Tous doivent faire face à l'inconnu. Les mécanismes et les stratégies d'adaptation qui sont déterminés par la culture, l'histoire, le statut social, la classe sociale, le genre, etc., peuvent varier d'un individu à l'autre. Les intervenants doivent comprendre l'impact de cela sur le processus d'intervention et développer une base de connaissances relatives à ces questions. Ainsi,

approfondir les déterminants macro-sociaux permet de saisir comment un même vécu de guerre, de violences, de torture, de fuite, de souffrance et d'exil contraint et influence les trajectoires d'insertion, les forces et les besoins des familles arrivant au Québec (Rachédi et Vatz-Laaroussi, 2004, p. 10).

La principale habileté à cultiver dans ce contexte est celle de l'écoute afin de tirer des leçons de l'expérience des réfugiés. Dans ce sens, les intervenants développent ce que Rachédi et Vatz-Laaroussi (2004) présentent comme le rôle d'accompagnateur. Les habiletés doivent tenir compte non seulement de l'origine ethnique, se portant à l'écoute du cadre de référence culturel du demandeur d'asile, mais aussi de l'expérience de la demande d'asile. C'est là que le rôle d'accompagnateur prend tout son sens, en redonnant aux demandeurs d'asile le pouvoir de la narration, c'est-à-dire le pouvoir de se dire et de raconter ce qu'ils ont vécu et comment ils vivent aujourd'hui leur situation. Les habiletés que les intervenants développent révèlent leur capacité à tirer des leçons d'une situation : « Des aidants efficaces permettent à ceux qu'ils aident de les renseigner quant à qui ou à quoi ils ont perdu et quant au sens de cette perte » (Williams, Zinner et Ellis, 1999, p. 5).

Les intervenants doivent aussi évaluer les habiletés fondamentales de facilitation, telles que savoir assister, répondre, réfléchir et interroger pour vérifier s'ils travaillent de manière adéquate auprès d'individus qui traversent une période de crise découlant de la précarité de leur statut. L'élaboration des objectifs devrait se fonder sur les besoins tels que les perçoit le demandeur.

\section{L'EXPÉRIENCE DE L'ASILE ET LES BESOINS}

Les réfugiés au sens de la Convention ont des besoins spéciaux qui découlent de leur expérience; certains pourraient connaître des troubles de stress post-traumatique (Lie, 2002; Rousseau 2000), de longues périodes de séparation de leur famille (Bertot et Mekki-Berrada, 1999), ainsi que la perte de leur pays, de leur statut social et de leur identité personnelle (Ward et Styles, 2003). « La perte est une thématique au cœur de la vie des réfugiés: la perte de leur langue, de valeurs culturelles, de rôles économiques, du respect, de statut social, d'un environnement familier, de religion et d'identité » (Sideris, 2003, p. 718). À cet égard, les réactions différentes au traumatisme selon la culture et les diverses manières dont les gens font face à la peine, à la douleur et à la perte sont des éléments importants à prendre en considération. 


\section{LA DÉFENSE DES DROITS}

Dans ce contexte, la défense des droits apparaît comme un mode d'intervention utile au cœur du travail auprès des demandeurs d'asile. Cambridge et Williams (2004) décrivent deux types de pratiques de défense des droits particulièrement importants.

Premièrement, l'intercession juridique. Cette forme de défense des droits « s'applique autant à la représentation juridique dans des cas civils et criminels qu'aux litiges et aux contrôles judiciaires ». Une bonne partie des interventions auprès de demandeurs d'asile consiste à s'occuper de questions juridiques. Les intervenants pourraient même être sollicités pour accompagner les demandeurs d'asile à leur audience relative à leur détention ou aux audiences de la Commission de l'immigration et du statut de réfugié. Pour l'intervenant, cela suppose une bonne compréhension des lois de l'immigration et des détails relatifs au processus décisionnel du statut de réfugié. Pour les demandeurs d'asile, trouver un avocat compétent est au cœur de la demande d'asile. La plupart des requérants ne comprennent pas le processus décisionnel, les différentes étapes qu'il comporte, ni ce qu'il leur faut faire pour assurer la bonne préparation de leur cas, ni comment se trouver un bon avocat. Cela suppose aussi que l'on se familiarise avec les ressources communautaires pour que le processus de présentation soit plus facile.

Deuxièmement, la défense des droits d'individus. L'empowerment se trouve au cœur de la défense des droits individuels. Pour les demandeurs d'asile, cela suppose qu'ils comprennent le système dans lequel ils se trouvent et qu'ils participent au développement de compétences qui leur accorderont une marge de manœuvre dans le cadre des possibilités d'action restreintes dont ils peuvent disposer. Par exemple, ils doivent comprendre qu'ils ont des options lors du choix de leur avocat, qu'ils peuvent changer d'avocat s'ils sentent qu'ils ne sont pas bien représentés et qu'ils peuvent le faire sans nuire à leur cause.

Enfin, le cas de Mohamed Cherfi a encore une fois porté la question des demandeurs d'asile sur la place publique. Ce cas a aussi soulevé des questions quant au rôle des églises pour ce qui est d'offrir un refuge à des demandeurs d'asile déboutés. Les églises ont soutenu que l'absence d'un processus d'appel adéquat dans le processus actuel d'établissement du statut de réfugié les oblige à offrir une protection contre un système défectueux. Cette question, ainsi que d'autres soulevées dans cet article, constitue la pierre d'assise de l'intervention auprès des demandeurs d'asile. Le travail social auprès de demandeurs d'asile doit être compris dans le cadre actuel de la mondialisation de la migration internationale et tenir compte des questions soulevées par l'arrivée de réfugiés au Canada. Assurer la protection des demandeurs d'asile et trouver réponse aux questions relatives à l'intégration et aux droits de citoyenneté sont des défis qui se posent à toutes les nations occidentales. Malgré les politiques restrictives mises en place pour refouler les mouvements de réfugiés, les situations politiques et sociales affligeantes qui surgissent dans différentes parties du monde font en sorte que le flot ne s'atténuera pas. 
Dans ce contexte, les compétences et les connaissances particulières à chacune des cultures constituent un niveau d'intervention auprès des demandeurs d'asile. Cependant, tout en étant conscients des enjeux propres à la culture et au pays d'origine, les intervenants doivent saisir le sens de la migration. La migration, qu'elle soit volontaire (immigrants) ou forcée (réfugiés et demandeurs d'asile), constitue une expérience douloureuse qui comporte toujours une rupture avec le passé. Le travail social peut jouer un rôle fondamental dans un processus qui sert à retisser les liens sociaux.

\section{RÉFÉRENCES BIBLIOGRAPHIQUES}

AdELMAN, H. (2002). « Canadian Borders and Immigration Post 9/11 », The International Migration Review, vol. $36, \mathrm{n}^{\circ} 1$, printemps, p. 15-28.

AIKEN, S. (2000). « Manufactoring "Terrorists": Refugees, National Security, and Canadian Law », Refuge: Canada's Periodical on Refugees, vol. 19, n 3, p. 54-73.

AldRige, F., et S. WAdDIngton (2001). Asylum Seekers' Skills and Qualifications Audit Pilot Project, NIACE (National Organization for Adult Learning).

AUstin, C., et D. ESTE (2001). «The working experiences of underemployed and refugee men », Canadian Social Work Review, vol. 18, n² 2, p. 213-229.

BARSkY, R.F. (2000). Arguing and Justifying: Assessing the Convention Refugees, Choice of Moment, Motive and Host Country, Aldershot, G.-B., Ashgate.

BEISER, M., et F. Hou (2001). « Language acquisition, unemployment and depressive disorder among Southeast Asian refugees: a 10-year study », Social Science and Medicine, vol. 53, $\mathrm{n}^{0} 10$, p. 1321-1334.

Beiser, M., S. Noh, F. Hou, V. Kaspar et J. Rummens (2001). "Southeast Asian refugees' perceptions of racial discrimination in Canada », Canadian Ethnic Studies, vol. 33, $\mathrm{n}^{\circ} 1$, p. 46-70.

Bertot, J., et A. Mekki-Berrada (1999). Des services aux demandeurs d'asile : Pourquoi ? Ce qu'en disent les intervenants d'organismes communautaires du grand Montréal, Table de concertation des organismes de Montréal au service des réfugiés, Montréal, Canada, rapport de recherche, septembre.

BLANC-ChalÉARD, M.-C. (2003). "Comment l'intégration devint une politique » PROASILE, Dossier thématique $\mathrm{n}^{\circ} 8$, L'intégration au-delà des faux semblants, 30 septembre, [En ligne]. [http://www.France-terre-asile.org/breve.php3?id_breve=4] (Consulté le 18 août 2004).

BLOCH, A., et L. Schuster (2002). «Asylum and welfare: contemporary debates », Critical Social Policy, vol. 22, n ${ }^{\circ}$ 3, août, p. 393-414.

CAmbridge, P., et L. Williams (2004). «Approaches to advocacy for refugees and asylum seekers: A development case study for a local support and advice service », Journal of Refugee Studies, vol. 17, $\mathrm{n}^{\circ} 1$, p. 97-113. 
CAstles, S., M. Korac, E. VAsta et S. Vertovec (2003). Integration: Mapping the Field, avec l'aide de Katrin Hansing, Fiona Moore, Emma NewCombe, Lucy Rix et Soojin Yu, rapport d'un projet réalisé par le Centre for Migration and Policy Research et le Refugee Studies Centre de I'University of Oxford pour le Immigration Research and Statistics Service (IRSS) du Home Office, décembre 2002, Home Office Online Report 28/03.

[http ://www.homeoffice.gov.uk/rds/pdfs2/rdsolr2803.doc].

CERE - CONSEIL EUROPÉEN SUR LES RÉFUGIÉS ET LES EXILÉS (septembre 1999). Position on the Integration of Refugees in Europe.

CHERAN, R. (2001). «Xeno racism and international migration », Refuge: Canada's Periodical on Refugees, vol. 19, $n^{\circ} 6$, août, p. 1-3.

CONSEIL CANADIEN POUR LES RÉFUGIÉS (CCR) (11 août 2004).

DANSO, R.K., et M.R. GRANT (2000). « Access to housing as an adaptive strategy for immigrant groups: Africans in Calgary », Canadian Ethnic Studies, vol. 32, $n^{\circ}$ 3, p.19-43.

Drozdek, B., A.K. Noor, M. LUtT et D.W. Foy (2003). « Chronic PTSD and medical services utilization by asylum seekers », Journal of Refugee Studies, vol. 16, n² 2, p. 202-211.

Hugo, G. (2002). « Australian Immigration Policy: The Significance of the Events of September $11 »$, International Migration Review, vol. $36, \mathrm{n}^{\circ}$ 1, p. 37-40.

KaZemipur, A., et S.S. Halli (2001). " Immigrants and new poverty: The case of Canada », International Migration Review, vol. 35, $\mathrm{n}^{\circ}$ 4, hiver, p. 1129-1156.

Krahn, H., T. Derwing, M. Mulder et L. WiLkinson (2000). « Educated and Underemployed: Refugee Integration into the Canadian Labour Market », International Journal of Migration and Integration, vol. 1, $\mathrm{n}^{\circ} 1$, p. 59-84.

KUNDNANI, A. (2001). «In a foreign land: the new popular racism », Race and Class, vol. 43, $\mathrm{n}^{0}$ 2, octobre-décembre, p. 41-60.

LACROIX, M. (2000). The Road to Asylum. Between Fortress Europe and Canadian Refugee Policy: The Social Construction of the Refugee Claimant Subjectivity, Montréal, thèse de doctorat à l'École de service social, Université McGill - Université de Montréal.

LACROIX, M. (2004). «The road to asylum. Canadian refugee policy and the social construction of the refugee claimant subjectivity: Understanding refugeeness », Journal of Refugee Studies, vol. 17, nº 2, p. 147-166.

Legault, G. (2000). L'intervention interculturelle, Montréal, Gaëtan Morin Éd.

LI, P.S. (2001). « Immigrants' propensity to self-employment: Evidence from Canada », International Migration Review, vol. 35, $\mathrm{n}^{\circ}$ 4, p. 1106-1128.

LIE, B. (2002). « A 3-year follow-up study of psychosocial functioning and general symptoms in settled refugees », Acta Psychiatrica Scandinavica, vol. 106, n 6, décembre, p. 415-425.

MARR, B., et P.L. SikLOS (2001). « Propensity of Canada's foreign-born to claim unemployment insurance benefits », The International Migration, vol. 39, $\mathrm{n}^{\circ} 1$, p. 89-110. 
Mestheneos E., E. IOANNIDI-Kapolou et S. Gaunt (1999). Bridges and Fences: Refugee Perceptions of Integration in the European Union, Groupe de travail sur l'intégration du CERE, Bruxelles, OCIV.

MeStheneOS, E., et E. IOANNIDI (2002). «Obstacles to refugee integration in the European Union member states », Journal of Refugee Studies, vol. $15, n^{\circ} 3$, p. 304-320.

Oxman-Martinez, J., et N. LapierRe-Vincent (2002). Precarious Immigration Status, Dependency and Women's Vulnerability to Violence: Impacts on Their Health, Centre d'études appliquées sur la famille, Université McGill et Immigration Metropolis (volet 4), Centre de recherche interuniversitaire de Montréal sur l'immigration, l'intégration et la dynamique urbaine.

PICKERING, S. (2001). "Common sense and original deviancy: News discourses and asylum seekers in Australia », Journal of Refugee Studies, vol. 14, $n^{\circ}$ 2, juin, p. 169-186.

RACHÉDI, L., et M. Vatz-LAAROUSSI (2004). « Favoriser la résilience des familles immigrantes par l'empowerment et l'accompagnement », Intervention, n 120, p. 6-15.

RenAUD, J., et L. GINGRAS (1998). Les trois premières années au Québec des requérants du statut de réfugié régularisé, Direction de la planification stratégique et Direction des communications du ministère des Relations avec les citoyens et de l'Immigration, gouvernement du Québec, coll. Études, recherches et statistiques, $n^{\circ} 2$, août.

RICHMOND, A.H. (2001). "Refugees and Racism in Canada », Refuge: Canada's Periodical on Refugees, vol. $19, \mathrm{n}^{\circ}$ 6, p. 12-20.

Rose, D., et B. RAY (2002). « Le logement des réfugiés à Montréal trois ans après l'arrivée : le cas des demandeurs d'asile ayant obtenu la résidence permanente », Revue de l'intégration et de l'immigration internationale, vol. 2, $n^{\circ} 4$, p. 455-492.

Rousseau, C., S. Moreau, A. Drapeau et C. Marotte (janvier 1997). Politique d'immigration et santé mentale : impact des séparations familiales prolongées sur la santé mentale des réfugiés, rapport présenté au Conseil québécois de la recherche sociale.

Rousseau, C., F. Crépeau, P. Foxen et F. Houle (2002). « The complexity of determining refugeehood: A multidisciplinary analysis of the decision-making process of the Canadian Immigration and Refugee Board », Journal of Refugee Studies, vol. 15, $n^{\circ}$ 1, p. 62-75.

SIDERIS, T. (2003). « War, Gender and Culture: Mozambican women refugees », Social Science and Medicine, vol. 56, $\mathrm{n}^{\circ}$ 4, p. 713-724.

Simmons, A.B. (2002). « Mondialisation et migration internationale : tendances, interrogations et modèles théoriques », Cahiers québécois de démographie, vol. 31, nº 1, p. 7-33.

SOYDAN, H. (1998). "Understanding migration », dans C. WILLIAMS, H. SOYDAN et M.R.D. JoHnSON (dir.), Social Work and Minorities, New York, Routledge, p. 20-35.

SOURANDER, A. (2003). «Refugee families during asylum seeking », Nordic Journal of Psychiatry, vol. 57, $\mathrm{n}^{\mathrm{0}} 3$, mai, p. 203-207.

VALTONEN, K. (2004). " From the margins to the mainstream: conceptualizing refugee resettlement », Journal of Refugee Studies, vol. 17, $\mathrm{n}^{\circ} 1$, p. 70-96. 
Van Selm, J. (2003). « Foreign Policy considerations in dealing with Afghanistan's Refugees: When security and protection collide », FMR, vol. 13, p. 16-18.

WARD, C., et I. STYLES (2003). «Lost and found: Reinvention of the self following migration », Journal of Applied Psychoanalytic Studies, vol. 5, $n^{\circ} 3$, p. 349-367.

WiLliams, M., E.S. ZinNeR et R. ElLIS (1999). « The connection between grief and trauma: An overview », dans E.S. ZINNER et M. WILLIAMS (dir.), When a Community Weeps: Case Studies in Group Survivorship, Philadelphie, Brunner/Mazdel, p. 3-17. 